\title{
Maximum Likelihood Estimation of the Kumaraswamy Exponential Distribution with Applications
}

\author{
K.A. Adepoju \\ University of Ibadan, ka.adepoju@ui.edu.ng \\ O. I. Chukwu \\ University of ibadan, unnachuks2002@yahoo.co.uk
}

Follow this and additional works at: http://digitalcommons.wayne.edu/jmasm

Part of the Applied Statistics Commons, Social and Behavioral Sciences Commons, and the Statistical Theory Commons

\section{Recommended Citation}

Adepoju, K. A. and Chukwu, O. I. (2015) "Maximum Likelihood Estimation of the Kumaraswamy Exponential Distribution with Applications," Journal of Modern Applied Statistical Methods: Vol. 14 : Iss. 1 , Article 18.

DOI: $10.22237 /$ jmasm/1430453820

Available at: http://digitalcommons.wayne.edu/jmasm/vol14/iss1/18

This Regular Article is brought to you for free and open access by the Open Access Journals at DigitalCommons@WayneState. It has been accepted for inclusion in Journal of Modern Applied Statistical Methods by an authorized editor of DigitalCommons@WayneState. 


\section{Maximum Likelihood Estimation of the Kumaraswamy Exponential Distribution with Applications}

\author{
K. A. Adepoju \\ University of Ibadan \\ Ibadan, Nigeria
}

\author{
O. I. Chukwu \\ University of Ibadan \\ Ibadan, Nigeria
}

The Kumaraswamy exponential distribution, a generalization of the exponential, is developed as a model for problems in environmental studies, survival analysis and reliability. The estimation of parameters is approached by maximum likelihood and the observed information matrix is derived. The proposed models are applied to three real data sets.

Keywords: Information matrix, Maximum likelihood, Moment generating function.

\section{Introduction}

A random variable $X$ has the exponential distribution if its cumulative distribution function for $x>0$ is given by

$$
F(x)=1-\ell^{-\lambda x}
$$

where $\lambda>0$ is a scale parameter, the probability density function is

$$
f(x)=\lambda \ell^{-\lambda x}
$$

Using the Kumaraswamy link function by Cordeiro and de Castro (2011) given as

$$
g(x)=a, b f(x)[F(x)]^{b-1}\left[1-F(x)^{b}\right]^{a-1}
$$

K.A. Adepoju is in the Department of Statistics. Email at ka.adepoju@ui.edu.ng. O.I. Chukwu is in the Department of Statistics. Email at unnachuks2002@yahoo.co.uk. 


\section{ADEPOJU \& CHUKWU}

By inserting (1) and (2) in (3) we have

$$
\begin{gathered}
g(x)=a, b \lambda \ell^{-\lambda \ell}\left(1-\ell^{-\lambda x}\right)^{b-1}\left[1-\left(1-\ell^{-\lambda x}\right)^{b}\right]^{a-1} \\
a, b, \lambda>0
\end{gathered}
$$

Another term of Kumaraswamy distribution can be obtained using the binomial series expansion. The Kumaraswamy exponential distribution in equation (4) can be expanded as follows:

$$
(1-m)^{K}=\sum_{j=1}^{K}(-1)^{j}\left(\begin{array}{l}
K \\
j
\end{array}\right) M^{j}
$$

as

$$
\begin{aligned}
g(x) & =a b \lambda \ell^{-\lambda x}\left(1-\ell^{-\lambda x}\right)^{b-1} \sum_{j=1}^{K}(-1)^{j}\left(\begin{array}{c}
K \\
j
\end{array}\right)\left(1-\ell^{-\lambda x}\right)^{b j} \\
& =a b \lambda \ell^{-\lambda x} \sum_{j=1}^{K}(-1)^{j}\left(\begin{array}{c}
K \\
j
\end{array}\right)\left(1-\ell^{-\lambda x}\right)^{b j+b-1}
\end{aligned}
$$

\section{Statistical inference}

Given a random variable $X$ following equation (4), the likelihood function is obtained as

$$
L=a^{n} b^{n} \lambda^{n} \ell \prod_{i=1}^{n} \ell^{-\lambda x}\left(1-\ell^{-\lambda x}\right)^{b-1}\left[1-\left(1-\ell^{-\lambda x}\right)^{b}\right]^{a-1}
$$

Taking log-likelihood of the above

$$
\begin{aligned}
\log L= & n \log a+n \log b+n \log \lambda-\lambda \sum_{i=1}^{n} x+(b-1) \sum_{i=1}^{n} \log \left(1-\ell^{-\lambda x}\right) \\
& +(a-1) \sum_{i=1}^{n} \log \left[1-\left(1-\ell^{-\lambda x}\right)^{b}\right]
\end{aligned}
$$




\section{MLE OF THE KUMARASWAMY DISTRIBUTION}

$$
\begin{aligned}
& \frac{\partial \log L}{\partial a}=\frac{n}{a}+\sum_{i=1}^{n} \log \left[1-\left(1-\ell^{-\lambda x}\right)^{b}\right] \\
& \frac{\partial \log L}{\partial b}=\frac{n}{b}+\sum_{i=1}^{n} \log \left(1-\ell^{-\lambda x}\right)-(a-1) \sum_{i=1}^{n} \frac{\left(1-\ell^{-\lambda x}\right)^{b} \log \left(1-\ell^{-\lambda x}\right)}{1-\left(1-\ell^{-\lambda x}\right)^{b}} \\
& \frac{\partial \log L}{\partial \lambda}=\frac{n}{\lambda}-\sum_{i=1}^{n} x+(b-1) \sum_{i=1}^{n} \frac{x \ell^{-\lambda x}}{1-\ell^{-\lambda x}}-(a-1) b \sum_{i=1}^{n} \frac{x \ell^{-\lambda x}\left(1-\ell^{-\lambda x}\right)^{b-1}}{1-\left(1-\ell^{-\lambda x}\right)^{b}}
\end{aligned}
$$

\section{Fisher information}

$$
\begin{aligned}
\frac{\partial^{2} \log L}{\partial a^{2}}= & -\frac{n}{a^{2}} \\
\frac{\partial^{2} \log L}{\partial b^{2}}= & -\frac{n}{b^{2}}-(a-1) \sum_{i=1}^{n} \frac{\left(1-\ell^{-\lambda x}\right)^{b}\left[\log \left(1-\ell^{-\lambda x}\right)\right]^{2}}{\left[1-\left(1-\ell^{-\lambda x}\right)^{b}\right]^{2}} \\
\frac{\partial^{2} \log L}{\partial \lambda^{2}}= & -\frac{n}{\lambda^{2}}-(b-1) \sum_{i=1}^{n} \frac{x}{\left(1-\ell^{-\lambda x}\right)^{2}}- \\
& b(a-1) \sum_{i=1}^{n} \frac{x^{2} \ell^{-\lambda x}\left(1-\ell^{-\lambda x}\right)^{b-1}}{1-\left(1-\ell^{-\lambda x}\right)^{b}}\left[\frac{(b-1) \ell^{-\lambda x}\left(1-\ell^{-\lambda x}\right)^{b-2}}{\left(1-\ell^{-\lambda x}\right)^{b-1}}-\frac{b \ell^{-\lambda x}\left(1-\ell^{-\lambda x}\right)^{b-1}}{\left(1-\ell^{-\lambda x}\right)^{b}}\right]
\end{aligned}
$$




\section{ADEPOJU \& CHUKWU}

$$
\begin{aligned}
& \frac{\partial^{2} \log L}{\partial a \partial b}=-\left(1-\ell^{-\lambda x}\right)^{b} \log \left(1-\ell^{-\lambda x}\right) \\
& \frac{\partial^{2} \log L}{\partial b \partial \lambda}=\sum_{i=1}^{n} \frac{x_{i} \ell^{-\lambda x_{i}}}{1-\ell^{-\lambda x_{i}}} \\
& \frac{\partial^{2} \log L}{\partial a \partial \lambda}=-b \sum_{i=1}^{n} \frac{x_{i} \ell^{-\lambda x_{i}}\left(1-\ell^{-\lambda x_{i}}\right)^{b-1}}{1-\left(1-\ell^{-\lambda x_{i}}\right)^{b}}
\end{aligned}
$$

\section{Application}

For the sake of numerical illustrations, the two data sets used by Raja and Mir (2011) are considered. The first data set is on the failure time of the conditioning system of an airplane and the second is the runs scored by a Cricketer in 27

\begin{tabular}{|c|c|c|c|c|c|c|}
\hline Min & $\mathbf{Q}_{1}$ & $\mathbf{Q}_{2}$ & Mean & $Q_{3}$ & Max & Var \\
\hline 1.0 & 12.5 & 22.0 & 59.6 & 83.0 & 261.0 & 5167.421 \\
\hline \multicolumn{2}{|c|}{ Skewness } & \multicolumn{2}{|c|}{ Kurtosis } & & & \\
\hline \multicolumn{2}{|c|}{1.693605} & \multicolumn{2}{|c|}{4.966655} & & & \\
\hline
\end{tabular}
innings at national level.

Table 1. Descriptive Statistics on Failure Time on Conditional System

Table 2. Descriptive Statistics in runs scored by a Cricketer

\begin{tabular}{lllllll} 
Min & $\mathbf{Q}_{1}$ & $\mathbf{Q}_{\mathbf{2}}$ & Mean & $\mathbf{Q}_{\mathbf{3}}$ & Max & Var \\
\hline 2.00 & 8.00 & 25.00 & 36.41 & 50.00 & 127.00 & 1149.02
\end{tabular}

\begin{tabular}{ll} 
Skewness & Kurtosis \\
\hline 1.124548 & 3.492725
\end{tabular}




\section{MLE OF THE KUMARASWAMY DISTRIBUTION}

Table 3. Failure Time on Conditional System

\begin{tabular}{|c|c|c|c|}
\hline \multirow[b]{2}{*}{ Model } & \multirow[b]{2}{*}{ Estimates } & \multicolumn{2}{|c|}{ Statistics } \\
\hline & & $\begin{array}{l}\text { Log- } \\
\text { likelihood }\end{array}$ & AlC \\
\hline Weibull & $\hat{\alpha}=0.8536, \hat{\lambda}=0.0183$ & -151.970 & 305.94 \\
\hline Lognormal & $\hat{\mu}=3.358, \hat{\lambda}=1.3190$ & 151.621 & 305.242 \\
\hline $\begin{array}{l}\text { Exponentiated } \\
\text { Weibull }\end{array}$ & $\hat{\alpha}=3.824, \quad \hat{\theta}=0.1732, \quad \hat{\delta}=82.235$ & -149.567 & 308.134 \\
\hline $\begin{array}{l}\text { Exponentiated } \\
\text { Gumbel }\end{array}$ & $\hat{\alpha}=1.9881, \hat{\lambda}=49.0638$ & -148537 & 299.074 \\
\hline $\begin{array}{l}\text { Exponentiated } \\
\text { Lognormal }\end{array}$ & $\hat{\alpha}=0.1542, \hat{\mu}=3.1353, \quad \hat{\delta}=0.3648$ & -148.659 & 303.318 \\
\hline $\begin{array}{l}\text { Lehman Type II } \\
\text { Exponential }\end{array}$ & $\hat{\alpha}=0.3439, \hat{\lambda}=0.0057$ & -152.6097 & 309.2593 \\
\hline Exponential & $\hat{\lambda}=0.0168$ & -152.6297 & 307.2593 \\
\hline $\begin{array}{l}\text { Kumaraswamy } \\
\text { Exponential } \\
\text { Distribution }\end{array}$ & $\hat{\alpha}=10.142, \quad \hat{b}=0.9129, \hat{\lambda}=0.0005$ & $-107 / 9653$ & 221.9306 \\
\hline
\end{tabular}

Table 4. Runs Scored by a Cricketer

\begin{tabular}{|c|c|c|c|}
\hline \multirow[b]{2}{*}{ Model } & \multirow[b]{2}{*}{ Estimates } & \multicolumn{2}{|c|}{ Statistics } \\
\hline & & $\begin{array}{l}\text { Log- } \\
\text { likelihood }\end{array}$ & AIC \\
\hline Gamma & $\hat{\alpha}=0.7235, \lambda=0.0127$ & -125.654 & 253.308 \\
\hline Weibull & $\hat{\alpha}=1.040, \lambda=36.985$ & -124.021 & 250.042 \\
\hline Lognormal & $\hat{\mu}=3.0534, \lambda=1.174$ & -125.059 & 252.118 \\
\hline $\begin{array}{l}\text { Exponentiated } \\
\text { exponential }\end{array}$ & $\hat{\alpha}=0.8126, \lambda=0.0153$ & -125.945 & 253.93 \\
\hline $\begin{array}{l}\text { Exponentiated } \\
\text { Lognormal }\end{array}$ & $\hat{\alpha}=0.578, \quad \hat{\mu}=3.1836, \quad \hat{\delta}=0.7834$ & -125.965 & 257.93 \\
\hline $\begin{array}{l}\text { Exponentiated } \\
\text { Gumbel }\end{array}$ & $\hat{\alpha}=1.873, \lambda=45.264$ & -124.843 & 251.686 \\
\hline Exponential & $\hat{\lambda}=0.0275$ & -124.0589 & 250.1177 \\
\hline $\begin{array}{l}\text { Kumaraswamy } \\
\text { Exponential }\end{array}$ & $\hat{\alpha}=0.13006, \hat{b}=0.9557, \hat{c}=0.00014$ & -108.7224 & 223.4449 \\
\hline
\end{tabular}




\section{ADEPOJU \& CHUKWU}

\section{Conclusion}

The probability density function of Kumaraswamy-exponential distribution was discussed and applied for two data sets. In first data set regarding failure times of the conditioning system of an aeroplane. Kumaraswamy exponential provided the best fit followed by exponentiated Gumbel. In second data set regarding run s scored by a cricketer Kumaraswamy exponential, Weibull and exponential distributions provided better fit.

\section{References}

Aarts, R. M. (2000). Lauricella functions. From Math World -- A Wolfram Web Resource, created by Eric W. Weisstein. http://mathworld.wolfram.com/LauricellaFunctions.html.

Akinsete, A., Famoye, F. \& Lee, C. (2008). The beta-Pareto distribution. Statistics, 42(6), 547-563. doi:10.1080/02331880801983876

Chaudhry, M. A. \& Zubair, S. M. (2002). On a class of incomplete gamma functions with applications. Chapman and Hall/CRC: Boca Raton, Florida.

Cooray, K. \& Ananda, M. M. A. (2008). A generalization of the half-normal distribution with applications to lifetime data. Communications in Statistics Theory and Methods, 37(9), 1323-1337. doi:10.1080/03610920701826088

Cordeiro, G. M \& de Castro, M. (2009). A new family of generalized distributions. Journal of Statistical Computation \& Simulation, 00(00), 1-17.

Cordeiro, G. M. \& Nadarajah, S. (2011). Closed-form expressions for moments of a class of beta generalized distributions. Brazilian Journal of Probability and Statistics, 25(1), 14-33. doi:10.1214/09-BJPS109

Eugene, N., Lee, C. \& Famoye, F. (2002). Beta-normal distribution and its applications. Communications in Statistics-Theory and Methods, 31(4), 497-512. doi:10.1081/STA-120003130

Exton, H. (1978). Handbook of hypergeometric integrals: Theory, applications, tables, computer programs. Halsted Press: New York.

Gupta, R. C., Gupta, P. L. \& Gupta, R. D. (1998). Modeling failure time data by Lehman alternatives. Communications in Statistics-Theory and Methods, 27(4), 887-904. doi:10.1080/03610929808832134 


\section{MLE OF THE KUMARASWAMY DISTRIBUTION}

Gupta, R. D. \& Kundu, D. (1999). Theory \& methods: Generalized exponential distributions. Australian and New Zealand Journal of Statistics, 41(2), 173-188. doi:10.1111/1467-842X.00072

Gupta, R. D. \& Kundu, D. (2001). Exponentiated exponential family: An alternative to gamma and Weibull distributions. Biometrical Journal, 43(1), 117-130. doi:10.1002/1521-4036(200102)43:1<117::AID-BIMJ117>3.0.CO;2-R

Raja, T. A. \& Mir, A. H. (2011). On extension of some exponentiated distributions with application. International Journal Of Contemporary Mathematical Sciences, 6(8), 393-400. 